\title{
Probing Rotation of Core-collapse Supernova with Concurrent Analysis of Gravitational Waves and Neutrinos
}

\author{
Takaaki Yokozawa ${ }^{1}$, Mitsuhiro Asano $^{1}$, Tsubasa Kayano ${ }^{2}$, Yudai Suwa ${ }^{3,4}$, \\ Nobuyuki Kanda ${ }^{1}$, Yusuke Koshioº ${ }^{2}$, and Mark R. Vagins ${ }^{5,6}$
}

\begin{abstract}
The next time a core-collapse supernova (SN) explodes in our galaxy, various detectors will be ready and waiting to detect its emissions of gravitational waves (GWs) and neutrinos. Current numerical simulations have successfully introduced multi-dimensional effects to produce exploding SN models, but thus far the explosion mechanism is not well understood. In this paper, we focus on an investigation of progenitor core rotation via comparison of the start time of GW emission and that of the neutronization burst. The GW and neutrino detectors are assumed to be, respectively, the KAGRA detector and a co-located gadolinium-loaded water Cherenkov detector, either EGADS or GADZOOKS!. Our detection simulation studies show that for a nearby supernova $(0.2 \mathrm{kpc})$ we can confirm the lack of core rotation close to $100 \%$ of the time, and the presence of core rotation about $90 \%$ of the time. Using this approach there is also potential to confirm rotation for considerably more distant Milky Way supernova explosions.
\end{abstract}

Subject headings: supernovae:general, neutrinos, gravitational waves, stars:rotation

\footnotetext{
${ }^{1}$ Graduate School of Science, Osaka City University, Sumiyoshi-ku, Osaka 558-8585, Japan

${ }^{2}$ Department of Physics, Okayama University, Okayama, Okayama, 700-8530, Japan

${ }^{3}$ Yukawa Institute for Theoretical Physics, Kyoto University, Oiwake-cho, Kitashirakawa, Sakyo-ku, Kyoto 606-8502, Japan

${ }^{4}$ Max-Planck-Institut für Astrophysik, Karl-Schwarzschild-Str. 1, 85748 Garching, Germany

${ }^{5}$ Kavli Institute for the Physics and Mathematics of the Universe (WPI), University of Tokyo Institutes for Advanced Study, University of Tokyo, Kashiwa, Chiba 277-8583, Japan

${ }^{6}$ Department of Physics and Astronomy, University of California, Irvine, Irvine, CA 92697-4575, USA
} 


\section{Introduction}

Supernova explosions are among the most energetic events in the universe. Core collapse supernovae are the final phase in the evolution of massive stars with $M \gtrsim 8 M_{\odot}$. Most of the released gravitational energy, $\sim 10^{53} \mathrm{ergs}$, is emitted as neutrinos and only a small fraction $\left(\sim 1 \%\right.$, so $\left.\sim 10^{51} \mathrm{ergs}\right)$ is used to produce the violent explosion itself (Bethe 1990). Although the historic detection of neutrinos from SN 1987A (Hirata et al. 1987; Bionta et al. 1987; Alekseev et al. 1987) validated our basic picture of core collapse (e.g. the total energy, the emission timescale of neutrinos, etc.), there are still large uncertainties concerning the explosion mechanism itself. In addition to photons and neutrino observations, the next nearby (probably galactic) supernova will also likely be observed using a relative newcomer to multi-messenger astronomy, namely gravitational waves (GWs). Owing to their weak coupling with matter they have the potential to provide us with information about the innermost part of these violent phenomena, and hence could serve as a unique window into the explosion mechanism (see Ott 2009; Kotake et al. 2012, and references therein). The new multi-messenger astronomy era will include observation of broad-band photons (from radio to gamma), multi-energy neutrinos (from $\mathrm{MeV}$ to $\mathrm{PeV}$ ), and multi-frequency GWs (from $\mathrm{Hz}$ to $\mathrm{kHz}$ ) using various telescopes and detectors already in operation as well as those coming online in the near future. Using these signals we can see different aspects of astronomical objects. What's more, after decades of effort recent developments in the numerical simulation of core-collapse supernovae are making remarkable progress: there are several simulation studies showing successful shock expansion (see Sec. 2.). In light of these facts, we will give some prospects of what we can learn from the next nearby core-collapse supernova, with special focus on the presence of progenitor core rotation.

We employ theoretical predictions of self-consistent signals of neutrinos and GWs based on recent simulations of multi-dimensional neutrino-radiation hydrodynamics (e.g., Suwa et al. 2010). By considering a realistic detector simulation that consists of not only responses for both GWs and neutrinos but also takes into account noise and statistical behavior, we study the feasibility of extracting information concerning the most crucial period in the death of massive stars. We define characteristic times: epoch time of $\mathrm{GW} \mathcal{T}_{G W}$ and of neutronization burst $\mathcal{T}_{\nu}$. Supposing that the core rotates rapidly, the GW would be detectable prior to neutronization burst (i.e. $\mathcal{T}_{G W}<\mathcal{T}_{\nu}$ ) and vice versa (see Pagliaroli et al. 2009; Halzen \& Raffelt 2009, for time determination of core bounce by neutrinos alone). This work is complementary to previous studies, in which a principal component analysis or Baysien analysis and many other approaches have been employed to extract infomation of core rotation from GW signals around core bounce alone (Havama et al. 2008; Dimmelmeier et al. 2007; Summerscales et al. 2008; Röver et al. 2009; Logue et al. 2012; Abdikamalov et al. 2014; Edwards et al. 2014; Engels et al. 2014, and many others). Since GW waveforms 
are significantly affected by the various initial conditions of simulations and status of GW detector, complex analyses are required if we use only GW. Instead, our method tackles this issue from a multi-messenger astronomical perspective; we simply compare the GW epoch and neutronization burst times. Our analysis has the potential to provide not only evidence of progenitor core rotation, but also the time of core bounce. By combining them, we can expect a more robust extraction of the rotation law and velocity of rotation.

For our reference GW detector we use KAGRA, which is currently under construction (Aso et al. 2013), and for our neutrino detector use either EGADS, which is now operating (Mori 2013), and GADZOOKS! (Beacom \& Vagins 2004), an upgraded version of Super-Kamiokande expected to start taking data in 2017. This is because (i) the supernova neutronization burst generates copious amount of electron-type neutrinos, making these gadolinium-loaded water Cherenkov detectors more suitable than the classic SuperKamiokande configuration filled with with ultrapure waten (Ikeda et al. 2007), and (ii) since KAGRA, EGADS, and GADZOOKS! are all located in the same place, i.e. the Kamioka mine, signal travel time between detectors does not have to be taken into account.

Section 2 describes the numerical simulation and supernova signals which are used in this analysis. Section 3 describes the characteristics of KAGRA, EGADS, and GADZOOKS!. Section 4 shows the method of time extraction of $\mathcal{T}_{G W}$ and $\mathcal{T}_{\nu}$, with the result of determining an estimator of progenitor core rotation. Finally, Section 5 contains a summary and discussion of our results.

\section{Numerical Simulation of Core-collapse Supernova}

In this section, we illustrate the numerical simulation of core-collapse supernova explosions. First, we shortly explain the basics of simulations. Secondly, numerical methods used in this study are given. Thirdly, hydrodynamic features are expressed, and finally neutrino and gravitational wave emissions are presented.

\subsection{Basics}

Since there are various types of physics involved in core-collapse supernova explosions, detailed numerical simulations are indispensable. For instance, gravity describes how the matter collapses and how much energy is released during the collapse. In addition, a final

\footnotetext{
${ }^{1}$ Here we neglect neutrino oscillation effects for simplicity.
} 
outcome of supernovae are neutron stars (NSs), so that the nuclear force, which determines the structure of a NS, is critically important. Neutrino interaction rates, which give the cooling rates of the core as well as heating rates of post-shock material by neutrino absorption (this will be explained later), are treated in great detail to give a quantitatively correct answer. This is because the total amount of neutrino emission is $\sim 10^{53} \mathrm{erg}$, while the explosion energy itself is $\sim 10^{51} \mathrm{erg}$, therefore only a few percent of energy deposition by neutrinos can drive the explosion; indeed, this is the so-called standard scenario of corecollapse supernovae (see Colgate \& White 1966; Bethe \& Wilson 1985 for original idea and Kotake et al. 2012; Janka 2012; Burrows 2013 for recent reviews).

Due to the complexity of the system, we have not achieved fully consistent explosions using simulation thus far. However, several exploding simulations arising from the neutrino heating mechanism have been reported in the last decade (Buras et al. 2006; Marek \& Janka 2009; Suwa et al. 2010; Müller et al. 2012; Bruenn et al. 2013; Pan et al. 2015 for 2D and Takiwaki et al. 2012; Melson et al. 2015; Lentz et al. 2015; Müller 2015 for 3D) 2 These simulations exhibited smaller explosion energy, i.e., $\sim 10^{49}-10^{50}$ ergs, than the observationally required value, $\sim 10^{51} \mathrm{ergs}$, leading to continuous accretion onto a NS beyond the maximum supportable mass, inevitably resulting in an eventual collapse to a black hole instead of a NS. More recently, although the small explosion energy problem remains, there has been a successful exploding simulation, which means the mass accretion onto a NS ceases and the final mass eventually settles into $\sim 1.3 M_{\odot}$, by Suwa (2014) using a progenitor with $11.2 M_{\odot}$ at zero-age main sequence phase. This explosion is a consequence of a steep density gradient between the $\mathrm{Si}$ and $\mathrm{O}$ layers, which results in a rapid decrease of the mass accretion rate onto the shock wave (Suwa et al. 2014). After this shell passes through the shock, the shock begins to expand and the system eventually produces the explosion. In this study, we use the same progenitor and the same simulation code.

\subsection{Methods}

The numerical methods used in this study are the same as Suwa et al. (2010, 2011, 2013, 2014); Suwa (2014). In this code, we solve neutrino-radiation hydrodynamics equations, which consist of hydrodynamics equations and neutrino radiative transfer equations (see Suwa et al. 2013 for more details).

\footnotetext{
${ }^{2}$ Note that some simulations of multi-dimensional neutrino radiation hydrodynamics also reported the failure of the explosion by neutrino heating (Burrows et al. 2006; Ott et al. 2008; Hanke et al. 2013; Dolence et al. 2015), so that this mechanism still contains ambiguities.
} 
In the hydrodynamics simulations we employ axial symmetry and Newtonian gravity. The neutrino transfer equations for $\nu_{e}$ and $\bar{\nu}_{e}$ are solved with an isotropic diffusion source approximation (IDSA) by Liebendörfer et al. (2009) and heavier leptonic neutrinos are not taken into account. The weak interaction rates are based on description of Bruenn (1985). In the current study, we neglect neutrino-electron scattering. The nuclear equation of state employed is Lattimer \& Swesty (1991) with an incompressibility parameter of $K=220 \mathrm{MeV}$. As for the initial condition, we employ $11.2 M_{\odot}$ model from Woosley et al. (2002), which was used in number of previous works.

The rotation is imposed via a shellular rotation law as (e.g. Eriguchi \& Mueller 1985)

$$
\Omega(r)=\Omega_{0} \frac{r_{0}^{2}}{r^{2}+r_{0}^{2}},
$$

where $\Omega(r)$ is an angular velocity with $r$ being the radius from the center, $\Omega_{0}$ is the angular velocity at the center, and $r_{0}$ is a radius that determines the degree of differential rotation. Here, we employ $r_{0}=1000 \mathrm{~km}$. In order to investigate how the rotation affects emissions of gravitational waves and neutrinos, we perform simulations with two different rotation strengths of $\Omega_{0}=0.0 \pi$ and $1.0 \pi$ radian $\mathrm{s}^{-1}$.

Since little is known about the rotation rates of central cores just prior to core collapse, we just employ these values. The latter one (period $P_{0}=2 \pi / \Omega_{0}=2 \mathrm{~s}$ ) is relatively faster than the current estimation based on stellar evolutionary calculations $\left(P_{0} \sim 100 \mathrm{~s}\right)$, which take into account angular momentum transfer processes (e.g., Heger et al. 2005). Note that these calculations contain many ambiguities for angular momentum transfer. The direct observational constraints for stellar core rotation have been given only for low-mass red giant stars using asteroseismology (Beck et al. 2012) and the core rotation rates of massive stars making supernovae are observationally still uncertain. The spin period distributions of young pulsars also give constraints on rotation of precollapse cores, which imply the core rotation periods prior to collapse are longer than several tens of seconds, if the angular momentum of the inner cores are conserved (e.g., Ott et al. 2006). On the other hand, subsequent GW emission induced by r-mode instability of NS may slow down spin of rapidly rotating (with almost breakup velocity) pulsars to period of $\sim 10 \mathrm{~ms}$, which is compatible with estimated birth periods of rapidly rotating pulsars like PSR J0537-6910 ( $P_{\text {pulsar }}=16.1$ $\mathrm{ms})$ and the Crab pulsar $\left(P_{\text {pulsar }}=33.5 \mathrm{~ms}\right)$ (Lindblom et al. 1998). Therefore, at present the core rotation profiles during the precollapse phase are highly uncertain, and the observation of GW can be a smoking gun of this important issue.

In this paper, we evaluate the gravitational wave emission from aspherical motion of fluids via the Newtonian quadrupole formulas of Mönchmeyer et al. (1991). In the axisymmetric case, the components of the dimensionless gravitational wave strain in the transverse- 
traceless (TT) gauge are represented as

$$
h_{\theta \theta}^{T T}=-h_{\phi \phi}^{T T}=\frac{1}{8} \sqrt{\frac{15}{\pi}} \sin ^{2} \alpha \frac{A_{20}^{E 2}}{D} \equiv h_{+},
$$

where $\alpha$ is the angle between the symmetric axis and the line of sight of the observer. In this study, we assume $\sin \alpha=1$ for simplicity. Due to the axial symmetry, the other component, $h_{\times}$, is vanishing. $A_{20}^{E 2}$ is a coefficient of the mass quadrupole contribution, which can be expressed by hydrodynamic variables as

$$
\begin{aligned}
A_{20}^{E 2}= & \frac{16 \pi^{3 / 2}}{\sqrt{15}} \frac{G}{c^{4}} \int_{-1}^{1} d \mu \int_{0}^{\infty} d r r^{2} \rho\left[v_{r}^{2}\left(3 \mu^{2}-1\right)+v_{\theta}^{2}\left(2-3 \mu^{2}\right)-v_{\phi}^{2}\right. \\
& \left.-6 v_{r} v_{\theta} \mu \sqrt{1-\mu^{2}}-r \partial_{r} \Phi\left(3 \mu^{2}-1\right)+3 \partial_{\theta} \Phi \mu \sqrt{1-\mu^{2}}\right]
\end{aligned}
$$

where $G$ is the gravitational constant, $c$ is the speed of light, $\mu=\cos \theta, \rho$ is the density, $v_{r}$, $v_{\theta}$ and $v_{\phi}$ are velocity vector components in $r, \theta$, and $\phi$ directions, and $\Phi$ is the gravitational potential. $\partial_{r}$ and $\partial_{\theta}$ are $\partial / \partial r$ and $\partial / \partial \theta$, respectively. $D$ it the distance between the observer and the source. We do not discuss the gravitational wave emission from anisotropic neutrino emission (Epstein 1978; Burrows \& Haves 1996; Mueller \& Janka 1997) because this component contributes to GW at later time and does not affect GW around the bounce time.

\subsection{Hydrodynamic Features}

After the simulation sets in, the gravitational collapse begins and the central density increases. Around $180 \mathrm{~ms}$, the central density reaches the nuclear density $\left(\sim 3 \times 10^{14} \mathrm{~g}\right.$ $\mathrm{cm}^{-3}$ ), which indicates the formation of a protoneutron star (PNS). The equation of state becomes much stiffer above this density so that the infall of material ceases and a shock wave is produced at the surface of the PNS. 3 The shock propagates outward initially, but loses its energy due to the photodissociation of iron and neutrino emission so that it decelerates. About $100 \mathrm{~ms}$ after the bounce, which is defined here by the time of the maximum central density, the shock expands again owing to the neutrino heating mechanism. All simulations used in this study result in explosion, i.e., we observe the shock expansion above a few

\footnotetext{
${ }^{3}$ The inner core mass at the bounce in our simulation is $\sim 0.7 M_{\odot}$. This is larger than the slowly rotating model in Dimmelmeier et al. (2008), in which they showed that the inner core masses for slowly rotating models range between $0.4-0.5 M_{\odot}$, depending on the progenitor models and equation of state. The difference is from omission of electron scattering, which lowers the electron fraction and leads to smaller effective inner core mass, and neglecting general relativistic effects in this study.
} 
thousands km. As for the nonrotating model, Suwa et al. (2013) and Suwa (2014) give more information, which are valid even for rotating models of this paper, since the rotation is not too strong to change the whole picture.

\subsection{Neutrinos and Gravitational Waves}

Figure 1 represents the time evolution of luminosities of electron-type neutrinos $\left(\nu_{e}\right.$; panel (a)) and antineutrinos $\left(\bar{\nu}_{e}\right.$; panel (b)), and their average energies. One can see the general trend does not depend on the initial rotation rate very much. In the first few tens of $\mathrm{ms}$ for $\nu_{e}$, there is the so-called neutronization burst, which is generated by the rapid absorption of electrons by protons (electron capture; $\mathrm{e}+\mathrm{p} \rightarrow \mathrm{n}+\nu_{e}$ ) in the regime between the shock and PNS, causing emission of large amounts of $\nu_{e}$. After that the neutrino luminosity is gradually decreasing, but a large luminosity is still observed. On the other hand, $\bar{\nu}_{e}$ does not have such a spiky structure due to lack of positrons before bounce. Both $\nu_{e}$ and $\bar{\nu}_{e}$ exhibit that the rotating model $\left(\Omega_{0}=1.0 \pi \mathrm{rad} \mathrm{s}^{-1}\right)$ results in slightly smaller luminosity due to stronger centrifugal force and slower contraction of the PNS. Therefore, we can argue that the currently employed rotation strength does not significantly change the generic picture about neutrinos. Note that we calculated neutrino luminosity at the outer boundary based on streaming particles of IDSA, a stationary-state solution of Boltzmann equation, so that the propagation time between emission site and the outer boundary is neglected. This approximation is fully consistent with the estimation of gravitational waves using the quadrupole formula.

Figure 2 depicts the time evolution of the gravitational wave signal. The gravitational

wave signals obtained in this study are all classified as type-I (Zwerger \& Mueller 1997; Dimmelmeier et al. 2008), which have a strong peak at the core bounce when the central density exceeds the nuclear density. Note that in the early phase the gravitational wave strength strongly depends on the initial rotation (see small panel). As for model without rotation, the density structure is almost spherically symmetric so that there is no GW emission. On the other hand, the strongly rotating model exhibits strong GW emission at the time of bounce because the centrifugal force makes the core asymmetric. Therefore, we can constrain the rotation strength by detecting GW at just the time of bounce. We can constrain the bounce time in turn using neutrino data as shown in Fig. 1, in which we show that the neutrino emission does not depend on the rotation strength so that neutrinos are a guaranteed signal from core-collapse supernova. 


\section{Detectors}

In this section, we introduce the KAGRA, EGADS, and GADZOOKS! detectors, which are used to determine progenitor core rotation in this paper.

As described in the introduction, one of the important benefits of employing is the close proximity of these three detectors. This makes it possible to avoid any significant timelag in arrival times of gravitational wave and neutrino signals due to distance between the detectors. In gravitational wave detection, the angular resolution of the source direction is not as good as that of optical telescopes, even in multiple-detector observations. According to the diffraction limit of wavelength $(\sim 300 \mathrm{~km}$ for $1 \mathrm{kHz})$ and interval of detectors $(\sim 1000 \mathrm{~km})$, typical angular resolution for the $\mathrm{kHz}$ band burst wave is only a few degrees (Grover et al. 2014). Water Cherenkov detectors have a similar, few-degree angular resolution for supernova explosions $\sim 10 \mathrm{kpc}$ away (i.e., near the galactic center) (Ando \& Sato 2002; Tomàs et al. 2003). These facts mean that the correction of arrival time based on direction for wellseparated detectors will have a larger error than the timing accuracy which is required in this paper study, $<1 \mathrm{msec}$, for neutrino and gravitational wave signals only. Closely placed detectors do not suffer from this problem.

\subsection{KAGRA Detector}

KAGRA is a laser interferometric gravitational wave detector which is being constructed in the Kamioka mine in Gifu, Japan (Kuroda \& the LCGT Collaboration 2010). The KAGRA interferometer consists of two 3-kilometer long laser cavity arms; all optical and vacuum systems are located inside a mountain, providing a silent and stable environment. KAGRA will employ cryogenic mirrors made of monolithic crystals of sapphire to reduce the thermal noise. Its target sensitivity is a few $\times 10^{-24}[1 / \sqrt{\mathrm{Hz}}]$ in strain ' $h$ ' of the space-time metric around $100 \mathrm{~Hz}$ (Aso et al. 2013). 4 There are other gravitational wave detectors which will have similar sensitivity, i.e. LIGO in the US (Abbott et al. 2009) and Virgo in Europe (Accadia et al. 2011). Their upgraded configurations 56 and a global observation network of four gravitational wave detectors are expected to be in operation in late 2017 or 2018 .

To achieve these detectors' high sensitivity various things are required: a long baseline

\footnotetext{
${ }^{4}$ KAGRA official sensitivity (http://gwcenter.icrr.u-tokvo.ac.ip/researcher/parameters)

${ }^{5}$ aLIGO ; https://www.advancedligo.mit.edu/

${ }^{6}$ aVirgo ; https://wwwcascina.virgo.infn.it/advirgo/
} 
to integrate strain effect, a high-power laser to protect against quantum shot noise, highquality and heavy mirrors, a vacuum system to eliminate air fluctuations, many advanced technique of optics including quantum optics, and so on. KAGRA employs additional special techniques, particularly in its underground site and cryogenic mirrors.

\subsection{EGADS and GADZOOKS! Detectors}

The EGADS (originally standing for Evaluating Gadolinium's Action on Detector Systems) detector (Mori 2013) is a gadolinium (Gd) loaded water Cherenkov detector initially built as a demonstrator for GADZOOKS! (Beacom \& Vagins 2004), the proposal to load Super-Kamiokande (SK) with a water-soluble gadolinium salt. EGADS is located in the Kamioka mine which is in the same mountain as both SK and KAGRA.

GADZOOKS! envisions adding $0.2 \%$ by mass of gadolinium sulfate $\left(\mathrm{Gd}_{2}\left(\mathrm{SO}_{4}\right)_{3}\right)$ into $\mathrm{SK}$ in order to facilitate the efficient detection of neutrons from Inverse Beta Decay (IBD) reactions $\left(\overline{\nu_{e}}+p \rightarrow e^{+}+n\right)$. Gadolinium has the highest capture cross section for thermal neutrons of any naturally occurring substance, and emits an 8.0 MeV gamma cascade following capture. This can be easily detected in water Cherenkov detectors like SK, making neutrons visible and thereby tagging up to $90 \%$ of the IBD events as genuine due to the coincidence in both time $(\sim 30 \mathrm{~ms})$ and space $(\sim 1$ meter $)$ between the prompt positron and the delayed gadolinium neutron capture cascade (Watanabe et al. 2009).

The efficient neutron tagging made possible by gadolinium loading brings many benefits to the venerable water Cherenkov technology in terms of supernova neutrino detection. First of all, the distinctive "gadolinium heartbeat" - the double pulse of positron Cherenkov light and neutron capture gamma cascade - from IBD events in a Gd-loaded detector will instantly identify any galactic supernova as genuine (Adams et al. 2013). Next, by allowing event-byevent tagging of the copious IBD events, a pure $\overline{\nu_{e}}$ time structure and energy spectrum can be precisely characterized and then subtracted away, exposing other, more subtle signals. Doing so yields a variety of powerful advantages, including: improving the determination of the supernova's position in the sky, measuring the temperature of the burst, spotting the moment of birth of a black hole, and potentially identifying the early neutronization burst.

The EGADS detector consists of a cylindrical stainless steel tank, whose height and radius are $6.7 \mathrm{~m}$ and $6.5 \mathrm{~m}$, respectively. A total of 240 inward-facing photodetectors line the inner walls of the EGADS tank. Most of these ( 90\%) are SK-style 50-cm diameter photomultiplier tubes (PMTs), while the rest are prototype light detectors of various sizes and designs being considered for use in the future Hyper-Kamiokande project (Abe et al. 
2011). The resulting active light collecting surface area in EGADS is $40 \%$, the same as in SK. The EGADS tank contains a total of 200 tons of Gd loaded water, about 100 tons of which is in front of the PMTs.

Following the R\&D phase of operations (2010-2014), EGADS has been repurposed as a dedicated supernova neutrino detector, with the acronym now standing for Employing Gadolinium to Autonomously Detect Supernovae. Deadtime-free front-end electronics (Nishino et al. 2009) have been purchased, the same type used in SK since 2008, and will be installed in EGADS in 2015. From that point on the detector will run continuously as a supernova neutrino detector with realtime online event reconstruction.

In addition to lacking effective neutron tagging in its current pure-water configuration,

SK cannot record all of the data produced by nearby supernovae (Yokozawa 2011). By contrast, EGADS not only already has highly efficient tagging but also full supernova sensitivity for the entire Milky Way galaxy; it would expect to record about 40 events for a core-collapse explosion at the galactic center and 100,000 events for an explosion at the distance of Betelgeuse. If and when GADZOOKS! goes forward, SK will of course gain the strong advantages of efficient neutron tagging - which will be even more powerful than in EGADS due to SK's much greater size - but SK's DAQ limitations will persist for very close bursts. For this reason, EGADS can be expected to continue to play a useful role as a supernova neutrino detector well into the future, whether or not gadolinium is added to Super-Kamiokande.

\section{Detection Simulation}

In this section we describe the detector signal simulation for KAGRA, EGADS, and GADZOOKS!, and the method of how to determine progenitor core rotation from these detectors. The analysis path is as follows: (i) Run supernova detection simulation for a given situation. (ii) Extract the epoch time of GW, $\mathcal{T}_{G W}$, and neutronization burst, $\mathcal{T}_{\nu}$. (iii) Compare these times and determine the presence of rotation or its absence. (iv) Loop 100,000 times and evaluate $P_{r}$, which is the probability of core rotation. The initial angular momentum of progenitor core rotation in the supernova models are given two different magnitudes: $\Omega_{0}=0.0 \pi$ and $1.0 \pi \mathrm{rad} \mathrm{s}^{-1}$. Four supernova scenarios are considered: uniform distributions of explosions at distances of $0.2 \mathrm{kpc}$ or $1.0 \mathrm{kpc}$, for which the detection simulations of KAGRA and the EGADS detector are used; and explosions at the galactic center or distributed throughout the galaxy, for which the detection simulations of KAGRA and GADZOOKS! are used. This distinction is made is because of serious doubts (as discussed in the EGADS/GADZOOKS! section) regarding the current SK DAQ system's performance at the extremely high rates expected for nearby explosions. The galactic center is defined as (right 
ascension, declination $)=\left(17 \mathrm{~h} 42 \mathrm{~m} 26.603 \mathrm{~s},-28^{\circ} 55^{\prime} 00.445^{\prime \prime}\right)$ and its distance is set to $10 \mathrm{kpc}$. The galactic distribution is generated by an exponential distribution model (Wainscoat et al. 1992), where the differential number of supernovae, $\mathrm{dN}$, is proportional to

$$
d n \propto R \cdot d R \cdot d z \cdot e^{-\frac{R^{2}}{2 R_{0}^{2}}} \cdot e^{-\frac{|z|}{h}},
$$

where $R_{0}$ and $\mathrm{h}$ are variation factors and

$$
\begin{array}{r}
R=3.5 \mathrm{kpc} \\
h=0.32 \mathrm{kpc} .
\end{array}
$$

Note that the incident direction strongly affects GW detector response, but has little bearing on neutrino detection.

\subsection{GW Analysis}

\subsubsection{Detector Signal}

The output signal of the interferometric detector, $s\left(t_{i}\right)$, will be sampled with finite frequency $1 / \delta t$, and can be written as

$$
s\left(t_{i}\right)=h\left(t_{i}\right)+n\left(t_{i}\right),
$$

where $i=0,1,2, .$. is sample index and $h\left(t_{i}\right)$ is gravitational signal from supernova with beam pattern, polarization of GW emission, and distance from the sources taken into account. The $16,384 \mathrm{~Hz}$ sampling frequency of the KAGRA detector is used. $n\left(t_{i}\right)$ is time domain of detector noise.

When generating $n\left(t_{i}\right)$, we assume stationary and Gaussian detector noise of the KAGRA detector. Figure 3 shows the sensitivity curve of bKAGRA detector (Aso et al. 2013).

The fluctuated noise in the frequency domain, $n_{s n}(f)$, has a Rayleigh distribution with mean value $\langle n(f)\rangle$. Applying an Inverse Fast Fourier Transform, the KAGRA noise signal in the time domain, $n_{\text {det }}\left(t_{i}\right)$, is obtained.

The detector's response for the long wavelength approximation, where the arm length of the detector is smaller than the reduced wavelength $\lambda / 2 \pi$, can be described with detector coordinates (latitude $\lambda$, longitude $L$, angle between East and bisector of the detector arms $\gamma$, angle between detector arms $\zeta$ ), GW source coordinates (right ascension $\alpha$, declination $\delta$, inclination angle from symmetric axis $\iota$ in $2 \mathrm{D}$ numerical simulation model), local sidereal 
time of the detector's site $\phi_{t}+\Omega_{r} t$, and polarization angle $\Phi$. These effects can be written as beam-pattern functions, $F_{+}, F_{\times}$. Using an axisymmetric explosion model, the inclination angle between $+Z_{w}$ axis should be taken into account. The detailed calculation is done in Jaranowski et al. (1998). In case of a gravitational wave emitted from the light source traveling in the $+Z_{w}$ direction, the signal $h$ can be written with two independent waves' polarizations. That is, the simulated detector signal is written as follows;

$$
s_{\text {det }}(t)=F_{+}\left(\alpha, \delta, \phi, \theta, \phi_{r}, t_{i}\right) h_{+}\left(r, \iota, t_{i}\right)+F_{\times}\left(\alpha, \delta, \phi, \theta, \phi_{r}, t_{i}\right) h_{\times}\left(r, \iota, t_{i}\right)+n_{\text {det }}\left(t_{i}\right) .
$$

The KAGRA detector's coordinates are summarized in Table 1.

\subsubsection{Calculation of Signal to Noise Ratio}

To evaluate signal and noise power from obtained $s_{\text {det }}(t)$, the Excess Power Filter (Anderson et al. 2001) and Short Time Fourier Transform (STFT) are used. The Excess Power Filter extracts signal power with given time $\left[t_{s}, t_{s}+\Delta t\right]$, where $t_{s}$ and $\Delta t$ are a start time and a time duration of STFT data, respectively, in a frequency band $\left[f_{s}, f_{s}+\Delta f\right]$. In this paper, the duration time $\Delta t$ is fixed at $31.25 \mathrm{~ms}$ and the frequency band is $[40,1000] \mathrm{Hz}$. We remove the peak frequency of thermal suspension noise when extracting signal power.

The simulated signal is whitened via a whitening filter to flatten the noise spectrum in the frequency domain. The whitened signal $\tilde{S}_{w}(f)$ is calculated by

$$
\tilde{S}_{w}\left(t_{s}, f\right)=\frac{\tilde{S}\left(t_{s}, f\right)}{\langle\tilde{N}(f)\rangle}
$$

and $\tilde{S}\left(t_{s}, f\right)$ is calculated by

$$
\tilde{S}\left(t_{s}, f\right)=\int_{t_{s}}^{t_{s}+\Delta t} s_{\text {det }}\left(t^{\prime}\right) W\left(t^{\prime}-t_{s}\right) \exp \left(-2 \pi i f t^{\prime}\right) d t^{\prime}
$$

where $\langle\tilde{N}(f)\rangle$ is obtained from the running median (Mohanty 2002) of simulated noise data, and $W(t)$ is a Hann window function.

The signal power $P_{s}$ and Signal to Noise Ratio (SNR) can be defined as follows:

$$
\begin{aligned}
P_{s}\left(t_{s}\right) & =\sqrt{\frac{\int \tilde{S}_{w}^{*}\left(t_{s}, f\right) \cdot \tilde{S}_{w}\left(t_{s}, f\right) d f}{\int\left\langle\tilde{N}_{w}^{*}(f) \cdot \tilde{N}_{w}(f)\right\rangle d f}}, \\
\operatorname{SNR}\left(t_{s}\right) & =\frac{P_{s}\left(t_{s}\right)-m}{\sigma}
\end{aligned}
$$


where $m$ and $\sigma$ are the normalized mean and deviation of $P_{n}=\sqrt{\int \tilde{N}_{w}^{*}(f) \cdot \tilde{N}_{w}(f) d f}$ distribution, respectively. To obtain $m$ and $\sigma$ we first evaluate the noise behavior, without injecting a supernova signal, and evaluate $P_{n}$. The noise distribution shows $m=1$ and $\sigma=0.14$.

\subsubsection{Extracting Start Time of GW Emission}

The center of the timing window which contains the first local maximum SNR is defined as $\mathcal{T}_{G W}$, the start time of GW emission. Figure 4 shows one example of the time variation of obtained SNR for each rotation model. The supernova distance is set to $10 \mathrm{kpc}$ and positioned in the optimal orientation for detection by the KAGRA detector. In this case, each supernova simulation gives $\mathcal{T}_{G W}$ values of $33 \mathrm{~ms}\left(0.0 \pi \mathrm{rad} \mathrm{s}^{-1}\right.$, blue $)$ and $-1.0 \mathrm{~ms}(1.0 \pi$ $\operatorname{rad~} \mathrm{s}^{-1}$, red), respectively.

We define the detection threshold for GW analysis as the first local maximum of the $\mathrm{SNR}>8$. This threshold corresponds to a False Alarm Ratio of about $\sim 10^{-6}$ per year.

Figures 5 and 6 show the $\mathcal{T}_{G W}$ distributions for each explosion rotation model for the four supernova scenarios being simulated: $0.2 \mathrm{kpc}$ (red) and $1.0 \mathrm{kpc}$ (blue) uniformly distributed, galactic center (green), and galactic distribution (magenta). The horizontal axis shows the time after core bounce, when the central progenitor core density becomes maximum. When the progenitor core is strongly rotating, $\mathcal{T}_{G W}$ is almost the same time as core bounce, with sharper distributions for closer supernova explosions. Unfortunately, due to the second peak of $h(t)$ in the $1.0 \pi \mathrm{rad} \mathrm{s}^{-1}$ model which can be found around $15 \mathrm{~ms}$ in Figure 2, some simulations show mis-identification of core bounce time.

\subsubsection{Model Dependence}

To evaluate the uncertainty of GW epoch extraction we apply the same analysis to supernova models provided by Dimmelmeier et al. (2008). Of these, we select the single centrifugal bounce models, which are marked with crosses in their Table III, because we are interested in fast rotating models. There are 25 models under this classification.

One example of the Dimmelmeier model for gravitational wave amplitude $h_{\text {Dim }}(t)$ and the time evolution of the maximum density $\rho_{\text {Dim,max }}(t)$ is shown in Fig. 7. This model is called 'e20b-ls'; the progenitor mass is $20 \mathrm{M}_{\odot}$ and the initial state is given by stellar evolution simulation.

Figure 8 shows the extracted time distributions when applying the above threshold to 
the 26 Dimmelmeier models. For all such models, the extracted times are within about $-1 \pm 2$ ms of our $1.0 \pi \mathrm{rad} / \mathrm{s}$ model. This result means that for strong GW models we can extract GW emission time with a few ms uncertainty.

\subsection{Neutrino Analysis}

We will now present our method for estimating time variation of the expected number of neutrino interactions and extraction of neutronization burst time.

As an aside, we would like to discuss the effect of neutrino mass on neutrino speed. If the neutrino mass is assumed to be $0.1 \mathrm{meV}$, derived from the current direct observational limit, cosmological limit (Komatsu et al. 2011), and neutrino-less double beta decay experiment limit (Gando et al. 2012), the latency versus light speed is of order $0.1 \mathrm{~ms}$ for an explosion at the center of the galaxy. Thus, the mass of neutrinos does not affect the following discussion.

As a further simplification to the analysis, we do not take neutrino oscillation effects into account, and only consider the electron flavor neutrino interactions while neglecting those of the $\mu$, and $\tau$ flavor neutrinos.

\subsubsection{Expected Number of Interactions}

From neutrino luminosity, $L_{\nu}(t)$, and mean energy, $\left\langle E_{\nu}(t)\right\rangle$ of Fig. 1, we obtained an energy distribution, $d n / d E(t)$, assuming a Fermi-Dirac distribution

$$
\begin{array}{r}
\left\langle E_{\nu}(t)\right\rangle=\frac{F_{3}}{F_{2}} T(t) \approx 3.15 T(t), \\
f(E, t)=\frac{E^{2}}{1+\exp (E / T(t))}, \\
\frac{d N}{d E(t)}=f(E, t) \times \frac{L_{\nu}}{F_{3} T^{4}\left(4 \pi r^{2}\right)},
\end{array}
$$

where $T(t)$ is absolute temperature for given time, $r$ is distance from Earth, and $F_{2}$ and $F_{3}$ is defined as

$$
\begin{aligned}
& F_{k}=\int_{0}^{\infty} \frac{x^{k}}{1+\exp (x)}, \\
& F_{2} \approx 1.803 \\
& F_{3} \approx 5.683 .
\end{aligned}
$$


To identify the time of the neutronization burst, the expected event rate must be obtained. We consider three types of interactions in the EGADS and GADZOOKS! detectors. The first one is electron neutrino-electron elastic scattering,

$$
\nu_{e}+e \rightarrow \nu_{e}+e
$$

This is the primary interaction with which to identify the neutronization burst. The cross section of the reaction is described in Bahcall et al. (1995). The second one is electron anti-neutrino elastic scattering,

$$
\overline{\nu_{e}}+e \rightarrow \overline{\nu_{e}}+e .
$$

The cross section is also described in Bahcall et al. (1995). Because this reaction occurs only in neutral current interactions, the cross section is six times smaller than that of electron scattering (Eq. 14), but this interaction will be a background of the neutronization burst search. The final one is inverse beta decay,

$$
\overline{\nu_{e}}+p \rightarrow e^{+}+n \text {. }
$$

The cross section is described in Totani \& Sato (1995). Gadolinium-loaded water Cherenkov detectors have the ability to separate inverse beta decay reactions from other reactions via tagging of the follow-on neutron. Because neutron tagging efficiency is roughly $90 \%, 10 \%$ of these interactions remain as background.

There are other events generated by supernova neutrinos in water Cherenkov detectors, such as (i) Neutrino-Oxygen interactions, (ii) $\mu, \tau$ neutrino-electron elastic scattering, and so on. But the rates of these interaction will be small, so that we do not take them into account in the following analysis. Also, because the duration of a neutronization burst is $\sim 10$ milliseconds, the detector background may also be ignored $\left(\sim 10^{-6}\right.$ events $\left.\mathrm{s}^{-1}\right)$.

Figure 9 shows the expected number of these three interactions in EGADS in the case of a supernova explosion near the center of our galaxy for the model with $\Omega_{0}=0.0 \pi \mathrm{rad} \mathrm{s}^{-1}$. Figure 10 shows the progenitor core rotation dependence of expected interaction rates. If the progenitor core is strongly rotating, the gravitational energy is converted to rotation energy, in which case the neutrino luminosity becomes smaller as compared with weaker rotation models. In the GADZOOKS! detector simulation, the volume of the detector is 225 times larger than the EGADS detector.

We employ Poisson statistics to represent the fluctuation of the observed number of neutrinos within $1 \mathrm{~ms}$ intervals. Figure 11 shows one example of the time fluctuation of observed neutrinos for for the model with $\Omega_{0}=1.0 \pi \mathrm{rad} \mathrm{s}^{-1}$. The distances and detectors considered are EGADS for explosions at $0.2 \mathrm{kpc}$ (red) and $1.0 \mathrm{kpc}$ (blue), and GADZOOKS! for explosions at $10 \mathrm{kpc}$ (green). 


\subsubsection{Extracting Neutronization Burst Time}

The neutronization burst time, $\mathcal{T}_{\nu}$, is obtained via the following steps: (i) Open $6 \mathrm{~ms}$ sliding window. (ii) Count the number of observed neutrinos. (iii) Shift $1 \mathrm{~ms}$ and calculate again. (iv) If maximum observed number of neutrinos exceeds three events, we define this to be an observation of the neutronization burst. (v) The center of the time window containing the maximum observed number of neutrinos is defined as $\mathcal{T}_{\nu}$. If there are multiple candidates for $\mathcal{T}_{\nu}$, the leftmost timing window is defined as $\mathcal{T}_{\nu}$. Figure 12 shows the maximum observed number of neutrinos for each model. As already shown in Fig. 10, the model dependence is small.

Figure 13 shows the $\mathcal{T}_{\nu}$ distributions for the $1.0 \pi \mathrm{rad} \mathrm{s}^{-1}$ model at $0.2 \mathrm{kpc}$ and $1.0 \mathrm{kpc}$ as seen by the EGADS detector, and at $10 \mathrm{kpc}$ and distributed throughout the galaxy as seen by the GADZOOKS! detector. For close supernova explosions, the resulting $\mathcal{T}_{\nu}$ distribution is quite narrow, and the $\mathcal{T}_{\nu}$ is estimated as expected. But as the supernova distance becomes greater, the number of observed neutrinos per burst becomes smaller, proportional to the usual $r^{-2}$ with $r$ being the distance. Therefore, the $\mathcal{T}_{\nu}$ distribution fluctuates, and statistical uncertainties become larger with increasing distance to the progenitor.

\subsection{Coincidence Analysis}

\subsubsection{Definition of Progenitor Core Rotation Estimator}

By comparing two parameters, $\mathcal{T}_{G W}$ and $\mathcal{T}_{\nu}$, we will be able to ascertain the probability that the progenitor's core was rotating at the time of collapse.

Figures 14 and 15 show the distributions of $\mathcal{T}_{G W}$ and $\mathcal{T}_{\nu}$ for the $0.0 \pi \operatorname{rad~s}^{-1}$ and $1.0 \pi$ $\mathrm{rad} \mathrm{s}^{-1}$ models, respectively. Except for a minor peak in the $\mathcal{T}_{G W}$ distribution for $1.0 \pi \mathrm{rad}$

$\mathrm{s}^{-1}$ model, a simple comparison between these two times seems to be enough for discussion of progenitor core rotation. So, the definition of progenitor core rotation is as follows: we calculate $t_{c}$ which is defined as

$$
t_{c}=\mathcal{T}_{G W}-\mathcal{T}_{\nu}
$$

and if $t_{c}<0$, we suppose progenitor core rotation, while for $t_{c}>0$, we suppose NO core rotation. 


\subsubsection{Results}

Tables 2 and 3 summarize the $P_{r}$ values for each progenitor core rotation model and scenario, where $P_{r}$ is defined as the estimator for core rotation when both GW and neutronization burst signals have been observed. From these $P_{r}$ values, we have obtained the following conclusions:

(1) For non-rotating model, $\left(0.0 \pi \mathrm{rad} \mathrm{s}^{-1}\right)$, the $P_{r}$ value is expected to be close to $0 \%$. In the cases with high observed neutrino statistics the $P_{r}$ value is indeed almost $0 \%$ as expected. But in the case of low neutrino statistics, the $P_{r}$ value becomes larger which leads in turn to reduced determination accuracy of the neutronization burst time $\left(\mathcal{T}_{\nu}\right)$ as shown in Fig. 13. (2) For the rapidly rotating model $\left(1.0 \pi \mathrm{rad} \mathrm{s}^{-1}\right)$, the $P_{r}$ value is, as expected, close to $100 \%$. But, as shown in Fig. 15, there are two peaks due to mis-identifying $\mathrm{T}_{G W}$. This makes for a lower $P_{r}$ for the more distant explosions. Still, even for the galactic cases, the $P_{r}$ value exceeds $70 \%$.

\section{Summary and Discussion}

By using a consistent supernova explosion model emitting both GWs and neutrinos, we investigate the progenitor core rotation to compare the GW emission start time, $\mathcal{T}_{G W}$, obtained from KAGRA detector, and neutronization burst time, $\mathcal{T}_{\nu}$, which is obtained from EGADS and GADZOOKS! detector. The results show if a nearby supernova is very close(in case of $0.2 \mathrm{kpc}$ ), we can correctly determine no or slow core rotation about $100 \%$ of the time if the progenitor core is indeed not or slowly rotating, and determine the presence of core rotation almost $100 \%$ if the progenitor core is rapidly rotated. But we investigate only nearby supernovae using only a single GW and neutrino detector for this analysis.

For future studies a coherent or coincidence analysis using multiple GW detectors would help to improve the detection efficiencies. Beyond these potential points for improvement, we currently investigate progenitor core rotation with only two parameters, $\mathcal{T}_{G W}$ and $\mathcal{T}_{\nu}$. Applying multiple classification analysis, the accuracy of $P_{r}$ is expected to be improved. These things represent our next homework.

Next, we comment on the limitations of our numerical model. First, we employed numerical results of two-dimensional (axisymmetric) Newtonian hydrodynamic simulation of a core-collapse supernova. It is well known that the hydrodynamic features between 2D

and 3D are different, especially for the cascade direction of turbulent motion (Hanke et al. 2012; Couch \& O'Connor 2014; Handy et al. 2014; Takiwaki et al. 2014). The forward cas- 
cade (from large-scale to small scale), which is typical in 3D case, may weaken the large scale prompt convection and increase the chance being classified as rapidly rotating. This aspect should be checked by performing 2D and 3D simulations with the same setups. More important limitations of this study are neglecting general relativistic (GR) and magnetohydrodynamic (MHD) effects. As for GR effects, there have been several GR simulations that include detailed microphysics (e.g. Dimmelmeier et al. 2008; Sekiguchi 2010; Ott et al. 2012; Müller et al. 2013; Kuroda et al. 2014), which implied that GR leads to higher characteristic frequencies of gravitational wave signals due to more compact protoneutron stars as a consequence of stronger gravity. As for MHD effects, the magnetic fields would transfer the angular momentum from inside to outside and a strong jet is launched if the initial magnetic fields are strong enough, which would modify the GW signatures (Obergaulinger et al. 2006; Scheidegger et al. 2010; Takiwaki \& Kotake 2011; Sawai et al. 2013). However, since MHD effects do not have a large impact around the bounce, our conclusion will not be affected even if we include magnetic fields in our simulation for future projects. Secondly, in this paper we employed one progenitor model, s11.2 of Woosley et al. (2002) because it is well studied by a number of previous works. It should be noted that the prompt convection depends on the structure of the progenitor model. Therefore, to assess the robustness of the results obtained in this study we need a more systematic study using multiple progenitor models. In addition, we employed one specific angular velocity distribution. More study of the dependence on the rotation profile is also needed (but see Abdikamalov et al. 2013). Thirdly, we employed simple weak interactions in solving Boltzmann equation of neutrinos, which may affect the convection driven by unstable configuration of lepton fraction (Lentz et al. 2012, e.g.,). Simulations with more detailed microphysics are needed. Finally, we did not take into account heavier leptonic neutrinos and neutrino oscillation effects. As described in Sec. 4.2.1, the elastic scattering cross sections for heavier leptonic neutrinos are about six times smaller than electron flavor neutrinos. If generated electron neutrinos are converted to other heavier flavor neutrinos, the height of the neutronization burst would be smaller than the present estimation and the detection efficiency of the neutronization burst would also be smaller. The conversion ratio depends on the neutrino mass hierarchy (normal or inverted), neutrino oscillation parameters (mixing angles and mass differences), relations between matter and self-interaction effects in the progenitor, and whether the neutrinos first pass through the Earth or not (Kachelrieß et al. 2005; Dighe \& Smirnov 2000)before being detected. In particular, the neutrino mass hierarchy is crucially important. In the normal hierarchy case, almost all of the electron neutrinos generated in the inner core will convert to heavier flavor neutrinos via the matter effect (neglecting self-interaction effects), and it would be difficult to apply the techniques used for this study. Even in the inverted hierarchy case, some electron neutrinos will be converted to heavier flavor neutrinos; this effect would 
not be negligible. This issue will also be investigated in a forthcoming paper.

\section{Acknowledgments}

This work was supported by the MEXT Grant-in-Aid for Scientific Research on Innovative Areas "New Developments in Astrophysics Through Multi-Messenger Observations of Gravitational Wave Sources" (Nos. 24103004, 24103005, 24103006, 25103511), JSPS postdoctoral fellowships for research abroad, MEXT SPIRE, and JICFuS. Numerical computations in this study were in part carried on XC30 at CfCA in NAOJ and SR16000 at YITP in Kyoto University.

\section{REFERENCES}

Abbott, B. P., Abbott, R., Adhikari, R., et al. 2009, Reports on Progress in Physics, 72, 076901

Abdikamalov, E., Gossan, S., DeMaio, A. M., \& Ott, C. D. 2013, ArXiv e-prints, arXiv:1311.3678

—. 2014, Phys. Rev. D, 90, 044001

Abe, K., Abe, T., Aihara, H., et al. 2011, ArXiv e-prints, arXiv:1109.3262

Accadia, T., Acernese, F., Antonucci, F., et al. 2011, Classical and Quantum Gravity, 28, 114002

Adams, S. M., Kochanek, C. S., Beacom, J. F., Vagins, M. R., \& Stanek, K. Z. 2013, ApJ, 778,164

Alekseev, E. N., Alekseeva, L. N., Krivosheina, I. V., \& Volchenko, V. I. 1987, 26, 237

Anderson, W. G., Brady, P. R., Creighton, J. D., \& Flanagan, É. É. 2001, Phys. Rev. D, 63, 042003

Ando, S., \& Sato, K. 2002, Progress of Theoretical Physics, 107, 957

Aso, Y., Michimura, Y., Somiya, K., et al. 2013, Phys. Rev. D, 88, 043007

Bahcall, J. N., Kamionkowski, M., \& Sirlin, A. 1995, Phys. Rev. D, 51, 6146 
Beacom, J. F., \& Vagins, M. R. 2004, Physical Review Letters, 93, 171101

Beck, P. G., Montalban, J., Kallinger, T., et al. 2012, Nature, 481, 55

Bethe, H. A. 1990, Reviews of Modern Physics, 62, 801

Bethe, H. A., \& Wilson, J. R. 1985, ApJ, 295, 14

Bionta, R. M., Blewitt, G., Bratton, C. B., Casper, D., \& Ciocio, A. 1987, Physical Review Letters, 58, 1494

Bruenn, S. W. 1985, ApJS, 58, 771

Bruenn, S. W., Mezzacappa, A., Hix, W. R., et al. 2013, ApJ, 767, L6

Buras, R., Janka, H., Rampp, M., \& Kifonidis, K. 2006, A\&A, 457, 281

Burrows, A. 2013, Reviews of Modern Physics, 85, 245

Burrows, A., \& Hayes, J. 1996, Physical Review Letters, 76, 352

Burrows, A., Livne, E., Dessart, L., Ott, C. D., \& Murphy, J. 2006, ApJ, 640, 878

Colgate, S. A., \& White, R. H. 1966, ApJ, 143, 626

Couch, S. M., \& O'Connor, E. P. 2014, ApJ, 785, 123

Dighe, A. S., \& Smirnov, A. Y. 2000, Phys. Rev. D, 62, 033007

Dimmelmeier, H., Ott, C. D., Janka, H.-T., Marek, A., \& Müller, E. 2007, Physical Review Letters, 98, 251101

Dimmelmeier, H., Ott, C. D., Marek, A., \& Janka, H.-T. 2008, Phys. Rev. D, 78, 064056

Dolence, J. C., Burrows, A., \& Zhang, W. 2015, ApJ, 800, 10

Edwards, M. C., Meyer, R., \& Christensen, N. 2014, Inverse Problems, 30, 114008

Engels, W. J., Frey, R., \& Ott, C. D. 2014, Phys. Rev. D, 90, 124026

Epstein, R. 1978, ApJ, 223, 1037

Eriguchi, Y., \& Mueller, E. 1985, A\&A, 146, 260

Gando, A., Gando, Y., Hanakago, H., et al. 2012, Phys. Rev. C, 86, 021601

Grover, K., Fairhurst, S., Farr, B. F., et al. 2014, Phys. Rev. D, 89, 042004 
Halzen, F., \& Raffelt, G. G. 2009, Phys. Rev. D, 80, 087301

Handy, T., Plewa, T., \& Odrzywołek, A. 2014, ApJ, 783, 125

Hanke, F., Marek, A., Müller, B., \& Janka, H.-T. 2012, ApJ, 755, 138

Hanke, F., Müller, B., Wongwathanarat, A., Marek, A., \& Janka, H.-T. 2013, ApJ, 770, 66

Hayama, K., Desai, S., Kotake, K., et al. 2008, Classical and Quantum Gravity, 25, 184022

Heger, A., Woosley, S. E., \& Spruit, H. C. 2005, ApJ, 626, 350

Hirata, K., Kajita, T., Koshiba, M., Nakahata, M., \& Oyama, Y. 1987, Physical Review Letters, 58, 1490

Ikeda, M., Takeda, A., Fukuda, Y., et al. 2007, ApJ, 669, 519

Janka, H.-T. 2012, Annual Review of Nuclear and Particle Science, 62, 407

Jaranowski, P., Królak, A., \& Schutz, B. F. 1998, Phys. Rev. D, 58, 063001

Kachelrieß, M., Tomàs, R., Buras, R., et al. 2005, Phys. Rev. D, 71, 063003

KAGRA official sensitivity. http://gwcenter.icrr.u-tokyo.ac.jp/researcher/parameters

Komatsu, E., Smith, K. M., Dunkley, J., et al. 2011, ApJS, 192, 18

Kotake, K., Takiwaki, T., Suwa, Y., et al. 2012, Advances in Astronomy, 2012, arXiv:1204.2330

Kuroda, K., \& the LCGT Collaboration. 2010, Classical and Quantum Gravity, 27, 084004

Kuroda, T., Takiwaki, T., \& Kotake, K. 2014, Phys. Rev. D, 89, 044011

Lattimer, J. M., \& Swesty, F. D. 1991, Nuclear Physics A, 535, 331

Lentz, E. J., Mezzacappa, A., Messer, O. E. B., et al. 2012, ApJ, 747, 73

Lentz, E. J., Bruenn, S. W., Hix, W. R., et al. 2015, ArXiv e-prints, arXiv:1505.05110

Liebendörfer, M., Whitehouse, S. C., \& Fischer, T. 2009, ApJ, 698, 1174

Lindblom, L., Owen, B. J., \& Morsink, S. M. 1998, Physical Review Letters, 80, 4843

Logue, J., Ott, C. D., Heng, I. S., Kalmus, P., \& Scargill, J. H. C. 2012, Phys. Rev. D, 86, 044023 
Marek, A., \& Janka, H. 2009, ApJ, 694, 664

Melson, T., Janka, H.-T., \& Marek, A. 2015, ApJ, 801, L24

Mohanty, S. D. 2002, Classical and Quantum Gravity, 19, 1513

Mönchmeyer, R., Schaefer, G., Müller, E., \& Kates, R. E. 1991, A\&A, 246, 417

Mori, T., ed. 2013, R \&D project for Gd-doped water Cherenkov detector

Mueller, E., \& Janka, H.-T. 1997, A\&A, 317, 140

Müller, B. 2015, ArXiv e-prints, arXiv:1506.05139

Müller, B., Janka, H.-T., \& Marek, A. 2012, ApJ, 756, 84

—. 2013, ApJ, 766, 43

Nishino, H., Awai, K., Hayato, Y., et al. 2009, Nuclear Instruments and Methods in Physics Research A, 610, 710

Obergaulinger, M., Aloy, M. A., \& Müller, E. 2006, A\&A, 450, 1107

Ott, C. D. 2009, Classical and Quantum Gravity, 26, 063001

Ott, C. D., Burrows, A., Dessart, L., \& Livne, E. 2008, ApJ, 685, 1069

Ott, C. D., Burrows, A., Thompson, T. A., Livne, E., \& Walder, R. 2006, ApJS, 164, 130

Ott, C. D., Abdikamalov, E., O'Connor, E., et al. 2012, Phys. Rev. D, 86, 024026

Pagliaroli, G., Vissani, F., Coccia, E., \& Fulgione, W. 2009, Physical Review Letters, 103, 031102

Pan, K.-C., Liebendörfer, M., Hempel, M., \& Thielemann, F.-K. 2015, ArXiv e-prints, arXiv: 1505.02513

Röver, C., Bizouard, M.-A., Christensen, N., et al. 2009, Phys. Rev. D, 80, 102004

Sawai, H., Yamada, S., \& Suzuki, H. 2013, ApJ, 770, L19

Scheidegger, S., Käppeli, R., Whitehouse, S. C., Fischer, T., \& Liebendörfer, M. 2010, A\&A, $514, \mathrm{~A} 51$

Sekiguchi, Y. 2010, Progress of Theoretical Physics, 124, 331 
Summerscales, T. Z., Burrows, A., Finn, L. S., \& Ott, C. D. 2008, ApJ, 678, 1142

Suwa, Y. 2014, PASJ, 66, L1

Suwa, Y., Kotake, K., Takiwaki, T., Liebendörfer, M., \& Sato, K. 2011, ApJ, 738, 165

Suwa, Y., Kotake, K., Takiwaki, T., et al. 2010, PASJ, 62, L49

Suwa, Y., Takiwaki, T., Kotake, K., et al. 2013, ApJ, 764, 99

Suwa, Y., Yamada, S., Takiwaki, T., \& Kotake, K. 2014, ArXiv e-prints, arXiv:1406.6414

Takiwaki, T., \& Kotake, K. 2011, ApJ, 743, 30

Takiwaki, T., Kotake, K., \& Suwa, Y. 2012, ApJ, 749, 98

—. 2014, ApJ, 786, 83

Tomàs, R., Semikoz, D., Raffelt, G. G., Kachelrieß, M., \& Dighe, A. S. 2003, Phys. Rev. D, 68, 093013

Totani, T., \& Sato, K. 1995, Astroparticle Physics, 3, 367

Wainscoat, R. J., Cohen, M., Volk, K., Walker, H. J., \& Schwartz, D. E. 1992, ApJS, 83, 111

Watanabe, H., Zhang, H., Abe, K., et al. 2009, Astroparticle Physics, 31, 320

Woosley, S. E., Heger, A., \& Weaver, T. A. 2002, Reviews of Modern Physics, 74, 1015

Yokozawa, T. 2011, International Cosmic Ray Conference, 4, 90

Zwerger, T., \& Mueller, E. 1997, A\&A, 320, 209 
Table 1: KAGRA detector coordinates.

\begin{tabular}{|c|c|}
\hline parameter & value \\
\hline \hline latitude of KAGRA detector $\lambda$ & $+36.41^{\circ}$ \\
\hline longitude of KAGRA detector $L$ & $+137.3^{\circ}$ \\
\hline angle between East and bisector of the detector arms $\gamma$ & $+75.0^{\circ}$ \\
\hline angle between detector arms $\zeta$ & $90.0^{\circ}$ \\
\hline
\end{tabular}

Table 2: The various scenarios' GW detection efficiencies (GW eff.), neutronization neutrino detection efficiencies (neutrino eff.) for (1) EGADS or (2) GADZOOKS!, their product, and the $P_{r}$ value for the $0.0 \pi \mathrm{rad} / \mathrm{s}$ model.

\begin{tabular}{|c|c|c|c|c|}
\hline Scenario & GW eff.[\%] & neutrino eff.[\%] & detection eff.[\%] & $P_{r}[\%]$ \\
\hline \hline 0.2kpc, uniform & 74.8 & $100.0(1)$ & 74.8 & 0.0 \\
\hline 1.0kpc, uniform & 46.5 & $46.8(1)$ & 21.9 & 20.8 \\
\hline Galactic Center & 0.0 & $97.5(2)$ & 0.0 & - \\
\hline Galaxy Dist. & 1.5 & $84.6(2)$ & 1.5 & 0.2 \\
\hline
\end{tabular}

Table 3: The various scenarios' GW detection efficiencies(GW eff.), neutronization neutrino detection efficiencies(neutrino eff.) for (1) EGADS or (2) GADZOOKS!, their product, and the $P_{r}$ value for the $1.0 \pi \mathrm{rad} / \mathrm{s}$ model.

\begin{tabular}{|c|c|c|c|c|}
\hline Scenario & GW eff.[\%] & neutrino eff.[\%] & detection eff.[\%] & $P_{r}[\%]$ \\
\hline \hline 0.2kpc, uniform & 88.0 & $100.0(1)$ & 88.0 & 98.4 \\
\hline 1.0kpc, uniform & 73.6 & $40.2(1)$ & 29.5 & 80.0 \\
\hline Galactic Center & 21.5 & $94.8(2)$ & 20.4 & 75.3 \\
\hline Galaxy Dist. & 26.7 & $81.7(2)$ & 24.7 & 76.2 \\
\hline
\end{tabular}




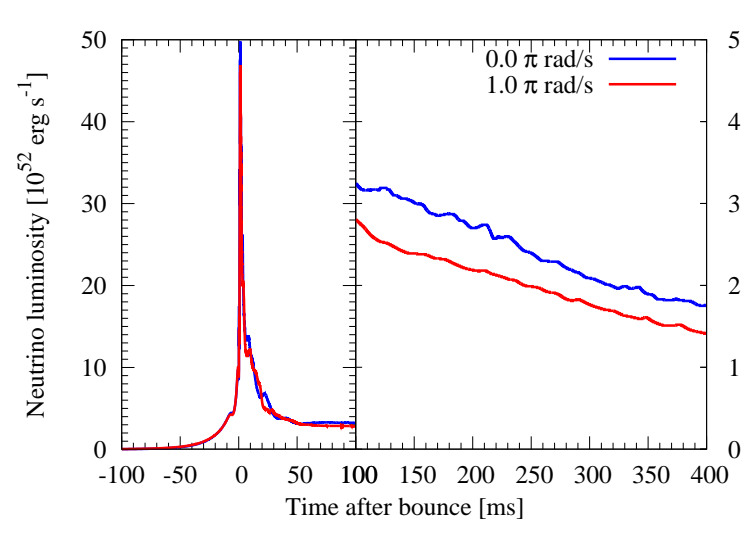

(a) $L_{\nu_{e}}$

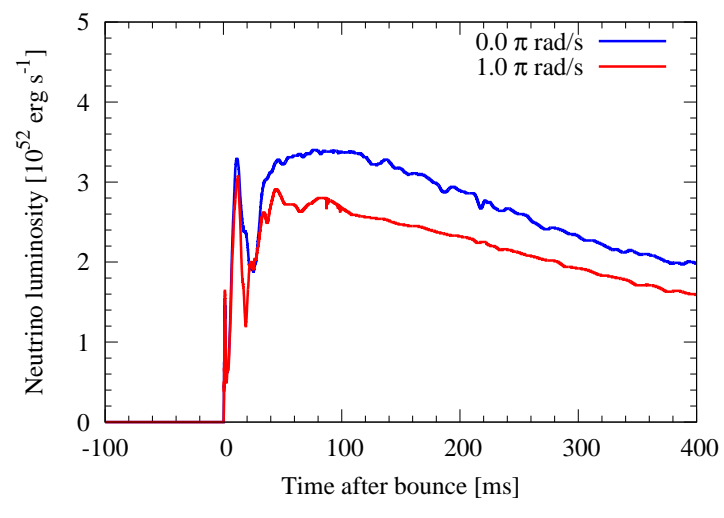

(b) $L_{\bar{\nu}_{e}}$

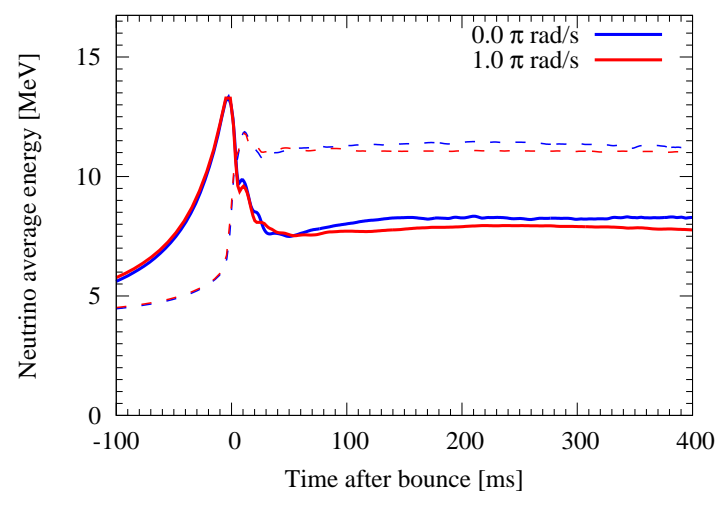

(c) $\left\langle E_{\nu}\right\rangle$

Fig. 1. - Time evolution of luminosities of electron-type neutrinos $\left(\nu_{e} ; \operatorname{panel}(\mathrm{a})\right)$ and antineutrinos $\left(\bar{\nu}_{e}\right.$; panel (b)) and average energy of them (panel (c)). Each line represents models with different initial rotation rates, i.e., no rotation $\left(\Omega_{0}=0.0 \pi\right)$ (blue) and strong rotation $\Omega_{0}=1.0 \pi \mathrm{rad} \mathrm{s}^{-1}$ (red). The solid and dashed lines in panel (c) show $\left\langle E_{\nu_{e}}\right\rangle$ and $\left\langle E_{\bar{\nu}_{e}}\right\rangle$, respectively. Note that left and right sides in panel (a) have different scales. 


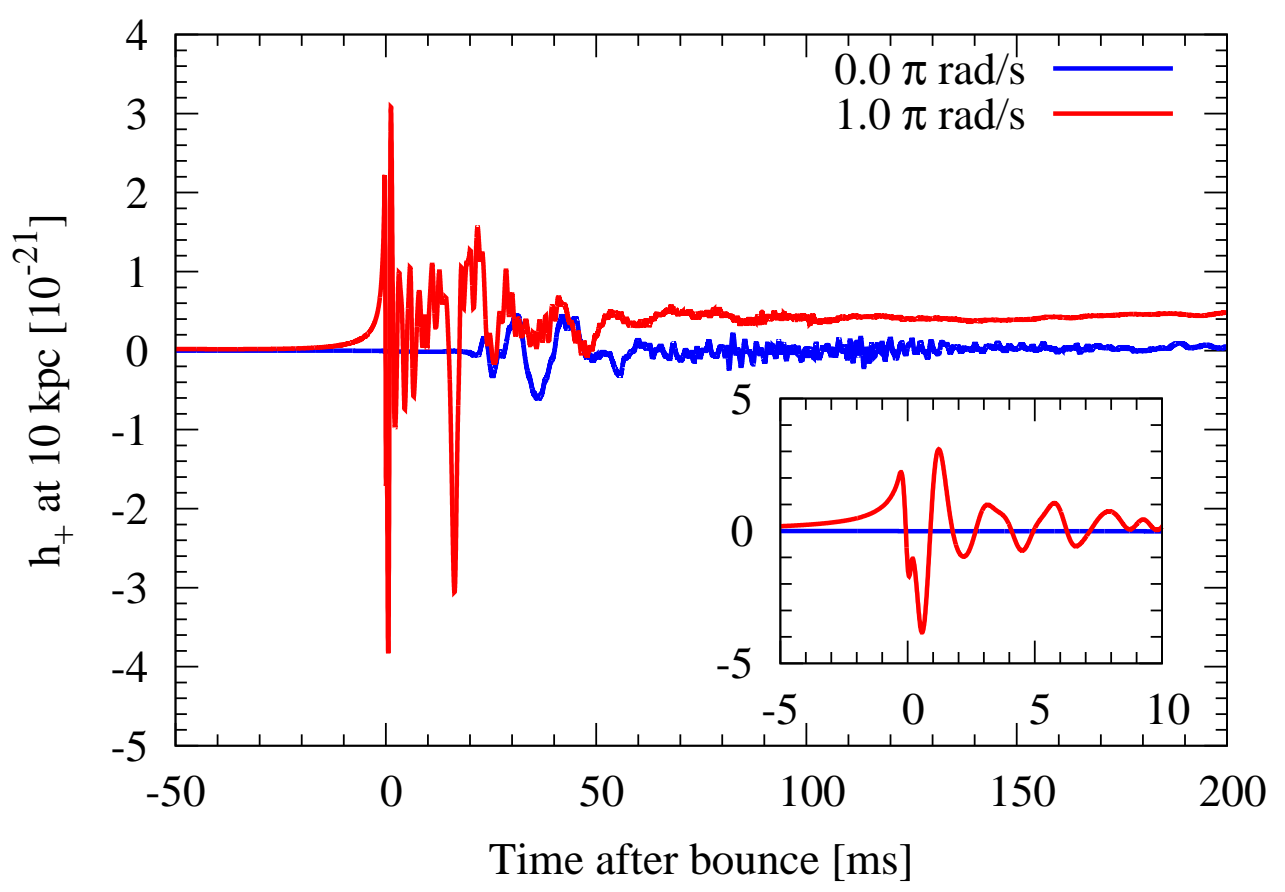

Fig. 2.- GW amplitude as a function of time for a core-collapse supernova occurring 10 kpc from the observer. Each line represents models with different initial rotation rate, i.e., no rotation $\left(\Omega_{0}=0.0 \pi\right)$ (blue) and strong rotation $\Omega_{0}=1.0 \pi \mathrm{rad} \mathrm{s}^{-1}$ (red). The small panel shows features around the bounce time. The fast rotation model exhibits a large amplitude GW at the bounce, while the slow rotation models have very small amplitudes. Since the later phase (i.e., a few tens of ms after the bounce) activity is dominated by convection motion, all models imply similar amplitude in the later phase. 


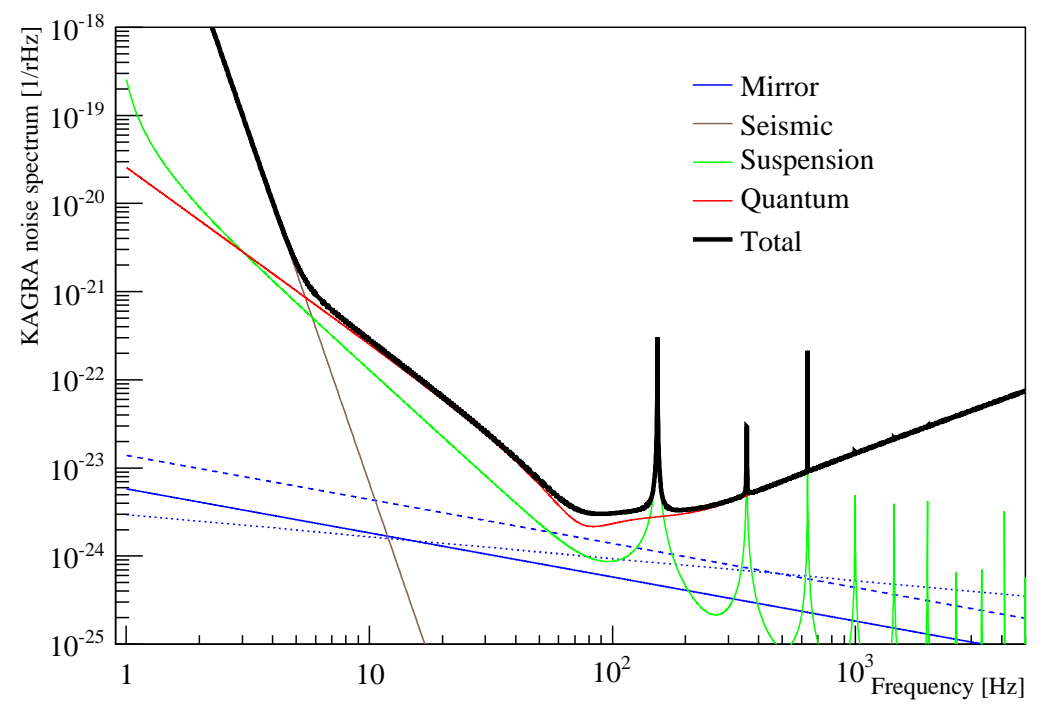

Fig. 3.- KAGRA official sensitivity curve. These curves are estimated from an incoherent sum of the fundamental noise sources. The colors show each type of environmental noise and the black line shows total noise. Blue lines are mirror related noise, brown shows seismic noise, green shows suspension thermal noise, and red shows quantum noise, respectively.

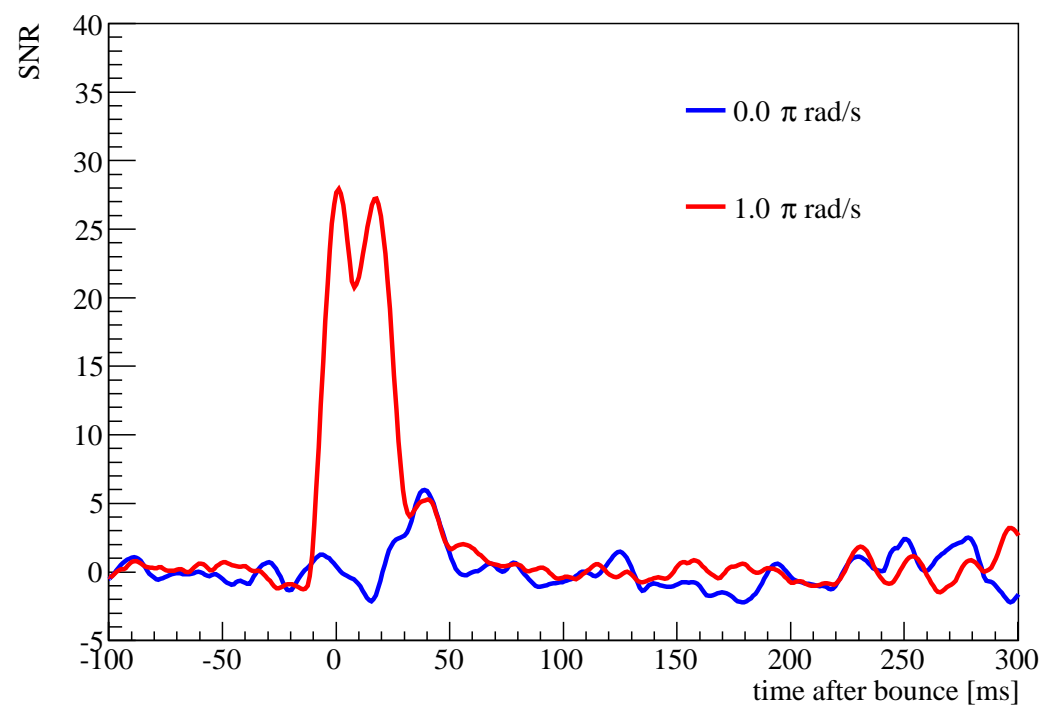

Fig. 4.- One example of the time variation of obtained SNR for each model. Each color shows one progenitor core rotation model, $0.0 \pi$ (blue) and $1.0 \pi$ (red) $\mathrm{rad} \mathrm{s}^{-1}$, respectively. The supernova distance is set to $10 \mathrm{kpc}$ and oriented for optimal detection by the KAGRA detector. 

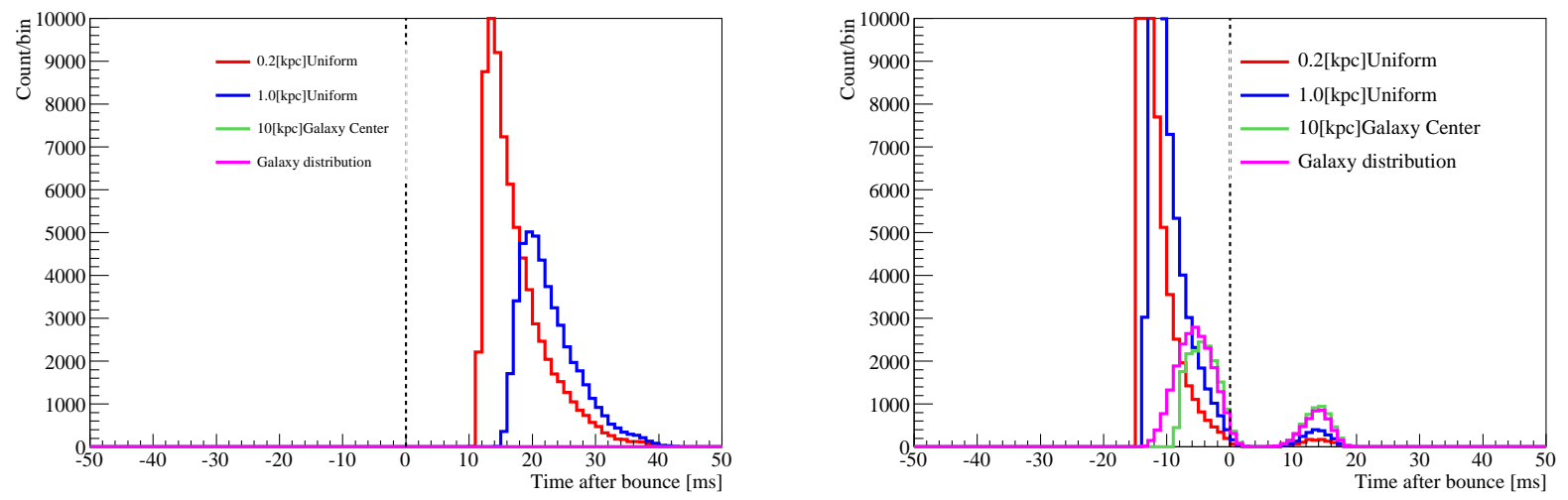

Fig. 5.- The $\mathrm{T}_{G W}$ extracted start time dis- Fig. 6. - The $\mathrm{T}_{G W}$ distribution of GW emistribution of GW emission for each explosion sion for each explosion model. The rotation model. The rotation is fixed at $0.0 \pi$ (red) rad $\mathrm{s}^{-1}$. Horizontal axis shows time from core shows time from core bounce. bounce.
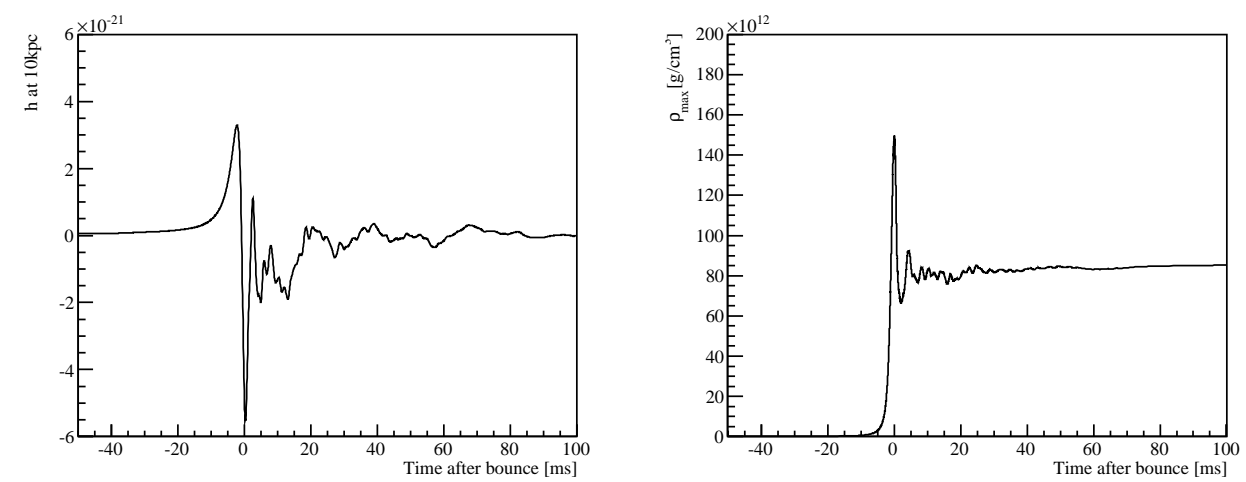

Fig. 7.- One example of the Dimmelmeier model, e20b-ls in the paper(Dimmelmeier et al. 2008). (Left) Time variation of gravitational wave amplitude $\mathrm{h}$ as a function of time from core bounce for $10 \mathrm{kpc}$. (Right) Time variation of maximum density $\rho_{\max }$. 


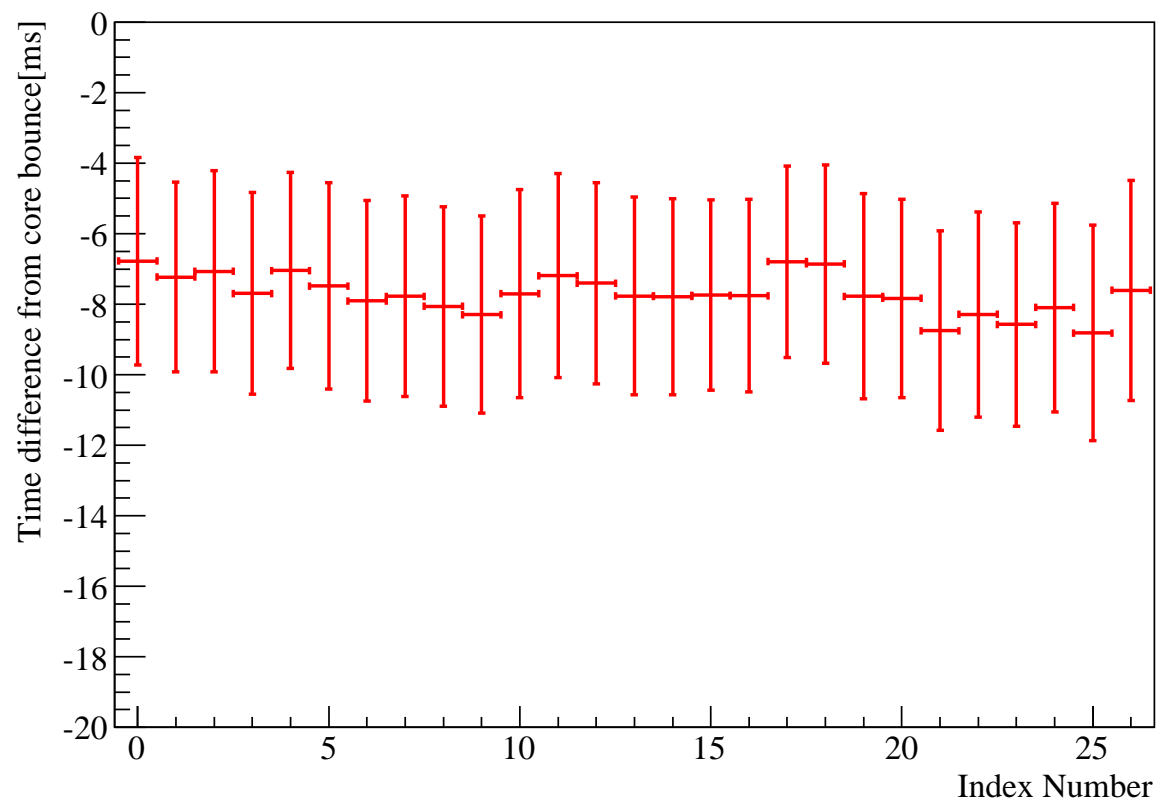

Fig. 8. - The DFM waveform dependence of the time difference from core bounce time for 26 Dimmelmeier models.

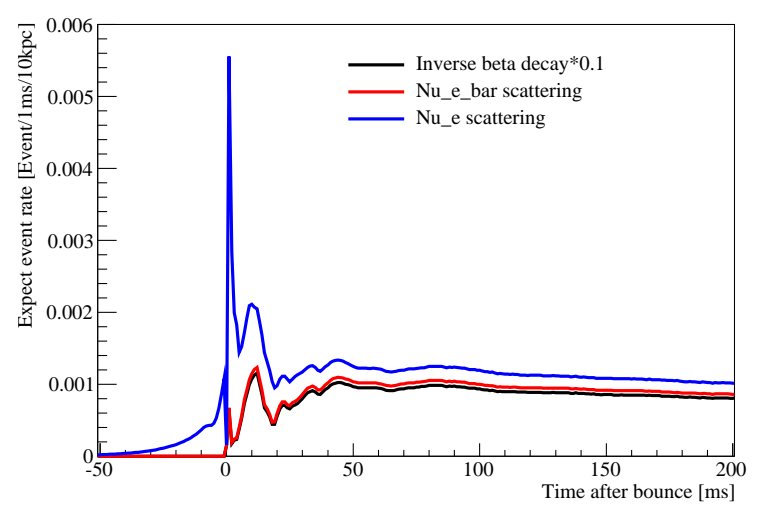

Fig. 9.- Expected number of interactions in EGADS. Black component shows electron neutrino-electron elastic scattering, blue component shows electron anti-neutrino-electron elastic scattering, and red component shows $10 \%$ of inverse beta decay interaction. Horizontal axis shows time and vertical axis shows unit of event $/ 1 \mathrm{~ms} / 10 \mathrm{kpc} / 100 \mathrm{ton}$.

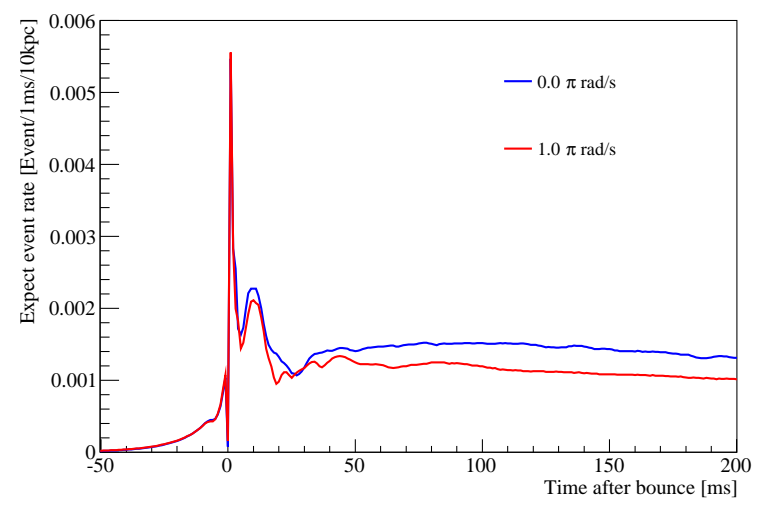

Fig. 10.- Model dependence of expected number of summed interaction in EGADS. Each color shows one progenitor core rotation model, $0.0 \pi$ (blue) and $1.0 \pi(\mathrm{red}) \mathrm{rad}$ $\mathrm{s}^{-1}$, respectively. Horizontal axis shows time and vertical axis is the sum of expected interactions in EGADS in units of event/1ms/10kpc/100ton. 


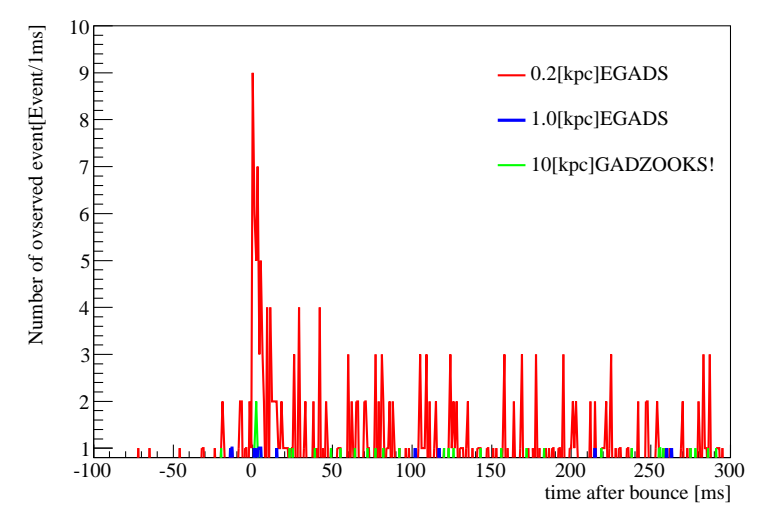

Fig. 11.- Fluctuation of the number of neutrinos observed by EGADS from distances of $0.2 \mathrm{kpc}$ (red) and $1.0 \mathrm{kpc}$ (blue), and by GADZOOKS! from a distance of $10 \mathrm{kpc}$ (green). The $1.0 \pi \mathrm{rad} \mathrm{s}^{-1}$ model is used for this figure.

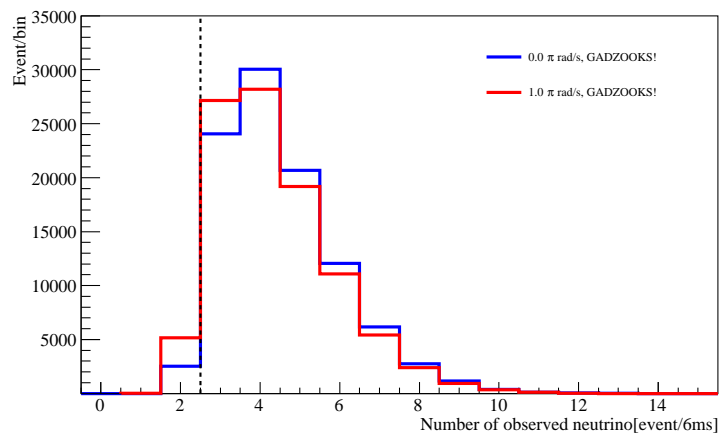

Fig. 12.- The number of maximum observed neutrinos used as a threshold. The GADZOOKS! detector and a burst at $10 \mathrm{kpc}$ are assumed. Colors show the progenitor core rotation models, $0.0 \pi$ (blue) and $1.0 \pi$ (red) rad $\mathrm{s}^{-1}$, respectively. Horizontal axis is number of observed events.

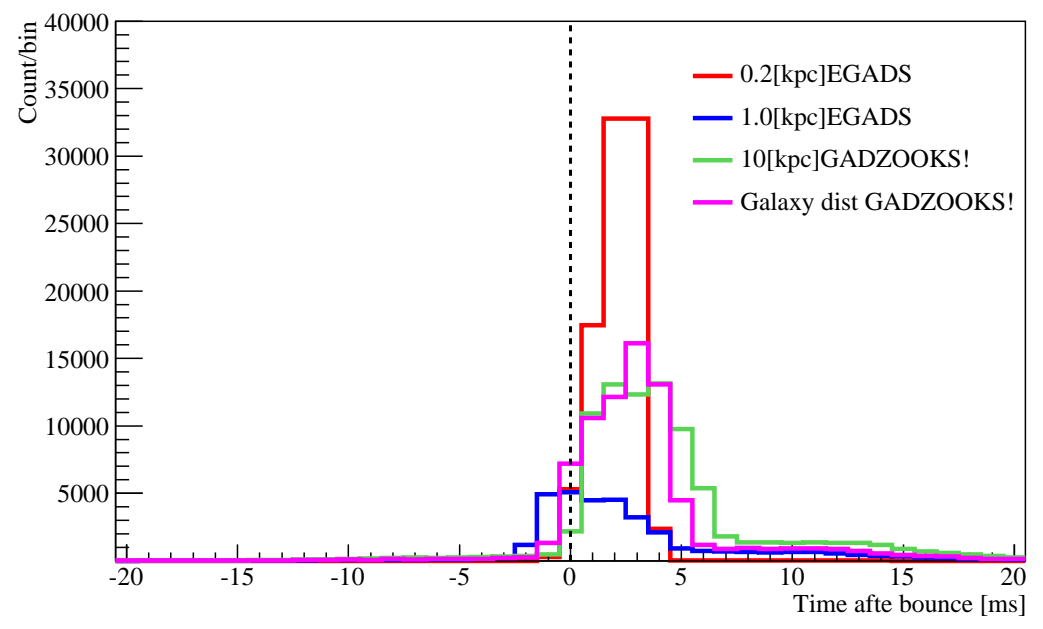

Fig. 13. - The obtained $\mathrm{T}_{\nu}$ distribution for $0.2 \mathrm{kpc}$ (red) and $1.0 \mathrm{kpc}$ (blue) with EGADS, and for $10 \mathrm{kpc}$ (green) and galactic distribution with GADZOOKS!. 

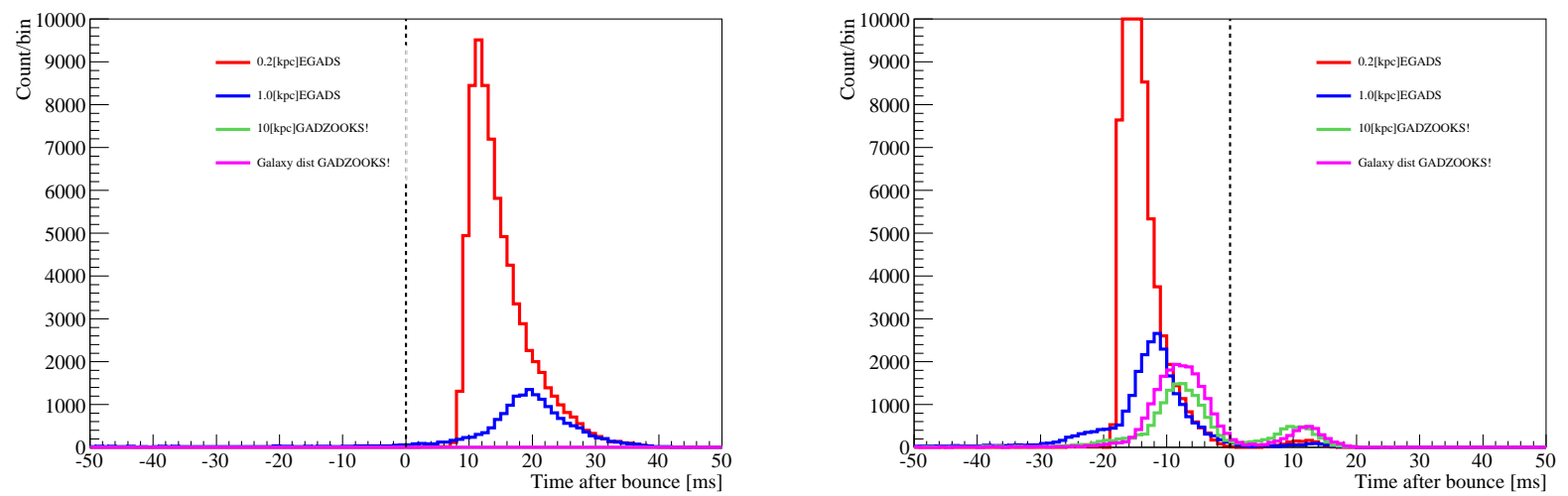

Fig. 14. - Time comparison between $\mathrm{T}_{G W}$ Fig. 15. - Time comparison between $\mathrm{T}_{G W}$ and $\mathrm{T}_{\nu}$ distribution for $0.0 \pi \mathrm{rad} \mathrm{s}^{-1}$ model. EGADS is used for $0.2 \mathrm{kpc}$ (red) and 1.0 kpc (blue), while GADZOOKS! is used for 10 $\mathrm{kpc}$ (green) and the galactic distribution (magenta). Both GW and neutrino distributions are made after applying detection threshold. are made after applying detection threshold. Horizontal axis shows time from core bounce. Horizontal axis shows time from core bounce. 